
cultures

Les cahiers de l'Acedle

16-1 | 2019

Enseigner la phonétique d'une langue étrangère

\title{
Médiation en didactique des langues vivantes.
}

Un exemple dans la formation des étudiants de l'ESPE de l'académie de Limoges.

Sophie Dufossé Sournin

\section{OpenEdition}

Journals

Édition électronique

URL : http://journals.openedition.org/rdlc/4681

DOI : $10.4000 /$ rdlc.4681

ISSN : 1958-5772

Éditeur

ACEDLE

Référence électronique

Sophie Dufossé Sournin, « Médiation en didactique des langues vivantes. », Recherches en didactique des langues et des cultures [En ligne], 16-1 | 2019, mis en ligne le 30 janvier 2019, consulté le 20 avril 2019. URL : http://journals.openedition.org/rdlc/4681; DOI : 10.4000/rdlc.4681

Ce document a été généré automatiquement le 20 avril 2019

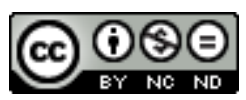

Recherches en didactique des langues et des cultures is licensed under a Creative Commons AttributionNonCommercial-NoDerivatives 4.0 International License 


\section{Médiation en didactique des langues vivantes.}

Un exemple dans la formation des étudiants de l'ESPE de l'académie de Limoges.

Sophie Dufossé Sournin

«Éduquer, c'est adapter l'individu au milieu social

ambiant. »

Piaget $(1969: 205)$

\section{Introduction}

1 L'apprentissage en autoformation est encouragé à l'université depuis plusieurs années maintenant. Ses potentialités sont souvent mises en avant afin de répondre à des exigences budgétaires, à des orientations pédagogiques ou à des développements technologiques. Qu'en est-il dans les parcours proposés par les Écoles supérieures du professorat et de l'éducation (désormais ESPE) ? Les technologies de l'information et de la communication pour l'enseignement (désormais TICE) représentent une discipline dispensée (soit le certificat informatique et internet de l'enseignement supérieur de niveau 2 ou C2i2e) et une compétence à valider pour enseigner dans les premier et second degrés en France. Cependant la préparation aux 2 concours du professorat que sont le concours de recrutement des professeurs des écoles (CRPE) et le certificat d'aptitude au professorat de l'enseignement du second degré (CAPES) semblent encore trop rarement s'appuyer sur des dispositifs pluriels, voire hybrides.

2 Dans ce compte-rendu d'expérience, nous proposons de décrire la mise en place d'un outil pédagogique lié à la formation des futurs enseignants. Ce matériel consiste en l'élaboration de modules didactiques expérimentés par les fonctionnaires-stagiaires et mis à disposition des étudiants préparant les épreuves orales du CAPES d'anglais sur la plateforme d'apprentissage en ligne Moodle ${ }^{1}$ de l'établissement. L'objectif est de dissocier temps et lieu de formation et d'optimiser cours en présentiel et en distanciel mais dans un dispositif qui prend en compte le matériel pédagogique déposé sur la plateforme de 
l'établissement. Il s'agit de rentabiliser les moyens existants plutôt que de multiplier les cours et donc le recrutement d'intervenants supplémentaires pour assurer des « colles » de fin d'année, par exemple.

3 Dans un premier temps, nous préciserons les motivations à l'origine d'un tel projet (demande des étudiants, constatations de l'enseignante, acteurs impliqués, enjeux). Nous décrirons ensuite la mise en place du dispositif en détaillant les différentes étapes nécessaires à son montage ainsi que les modifications apportées au fur et à mesure de sa mise en place. Enfin, nous nous interrogerons sur le rôle de l'enseignante dans un projet relevant d'une pédagogie « partagée » en termes de qualité, d'efficacité et de pérennité.

\section{Observation}

4 Ce projet relève de la didactique des langues vivantes avec, pour origine, un besoin spécifique exprimé par les candidats de Master $1{ }^{\text {ère }}$ année « Métiers de l'enseignement, de l'éducation et de la formation » (désormais MEEF) de l'ESPE, parcours second degré, de la XXX. Plus précisément, une demande précise des représentants des étudiants ${ }^{3}$, lors du dernier conseil de perfectionnement de l'année universitaire 2015-2016, nous a incitée à créer un outil pédagogique au service de tous. Il était alors question de rencontrer les fonctionnaires-stagiaires de Master $2^{\text {ème }}$ année afin d'échanger sur leur pratique pédagogique. Nous avons alors constaté que les inscrits en Master MEEF ne se connaissaient pas, et que l'expérience vécue par les M2 pouvait être bénéfique aux M1. Nous avons donc envisagé une médiation entre M1 et M2. Elle a pris la forme d'un outil didactique utile à la préparation au concours de l'enseignement secondaire pour l'année universitaire 2016-2017. Il s'agissait également de dissocier cet entraînement du temps et du lieu de formation habituels, dans le but de rentabiliser du matériel plutôt que de multiplier les sources d'interventions enseignantes impossibles aujourd'hui, faute de moyens. En effet, l'université n'envisage pas de recruter d'intervenants supplémentaires. Lamy (2011: 614) le rappelle, « [...] "produire plus [de produits éducatifs] pour enseigner plus" ne constitue pas une bonne réponse à la pression budgétaire. "Produire mieux en restant au diapason des tendances sociétales" fait en revanche partie des bonnes réponses. »

5 La mise en œuvre de ce dispositif d'apprentissage a pour but précis la préparation aux épreuves orales d'admissibilité au CAPES d'anglais. L'objectif de l'enseignement d'une langue vivante aujourd'hui est de développer une compétence de communication susceptible de rendre l'utilisateur indépendant et autonome dans sa pratique personnelle et professionnelle. Plus précisément, il s'agit de proposer du matériel utilisable pour la préparation à l'épreuve de mise en situation professionnelle (désormais MSP) élaboré par les néo-diplômés ayant encore en mémoire les besoins en termes de savoir et savoir-faire nécessaires à la réussite au concours. Cet examen oral demande des connaissances pédagogiques relevant globalement de l'expérience d'enseignement sur le terrain. L'épreuve de MSP se divise en 2 parties ${ }^{4}$. La première, appelée « synthèse » est purement universitaire. Son objectif est de mettre en relation un ensemble de plusieurs documents en anglais afin d'en dégager une problématique autour d'un point de langue ou de civilisation, par exemple, et de la développer selon les notions au programme. La seconde, appelée didactique, exige des étudiants qu'ils proposent une exploitation du dossier présenté lors de la première épreuve, selon un cycle d'enseignement visé imposé5. Pour ce faire, ils doivent prendre appui sur les documents à leur disposition et les organiser en 
fonction de leur spécificité et de leur potentiel en répondant aux exigences du public concerné, du rythme scolaire d'aujourd'hui, ainsi que des programmes et Instructions Officielles en vigueur. L'objectif est de favoriser une mise en œuvre pédagogique utile à l'enseignement-apprentissage de la langue-culture, dans le cadre de la construction de la compétence de communication. Les tâches à exécuter doivent être claires et mener à l'accès au sens des documents tout en amenant l'élève à " un degré supplémentaire d'autonomie » (Pinéda, 2016 : 73). Bailly (1997: 85) a expliqué [qu']« Un certain nombre d'habitudes cognitives, de pensées et de traitement de données, peuvent se mettre en place graduellement, permettant en principe à chaque élève de travailler plus rationnellement, de façon plus indépendante.»Dewey, (1976:143) proposait déjà que l'on fasse « construire et produire, au lieu de faire avant tout absorber et apprendre. »

Les étudiants de M1 ayant effectué 2 stages massés ${ }^{6}$ relevant de l'observation de pratiques de classe, manquent parfois du recul nécessaire pour concevoir des progressions et des activités cohérentes, correspondant aux objectifs visés par le dossier proposé lors de l'épreuve orale de MSP. Les évaluations faites dans le cadre du contrôle continu à l'ESPE montrent souvent un manque de cohérence entre les exploitations pédagogiques proposées et la réalité de terrain mais également entre le développement des activités langagières et les objectifs définis. Ces difficultés sont une source de stress tout au long de l'année universitaire. Notre dispositif a pour vocation de :

- proposer une formation pré-professionnelle optimisée venant enrichir l'enseignement en présentiel;

- développer une aide à la préparation au concours de l'enseignement secondaire compétitive par rapport aux grandes universités régionales;

- renforcer et justifier l'offre de formation initiale (voire continue dans les années à venir), proposée à Limoges et conforter les résultats obtenus jusqu'à présent dans la région ;

- collaborer à la refonte des maquettes d'offre de formation de l'ESPE de l'académie de XXX en respectant le cahier des charges imposé.

7 Ce projet s'inscrit dans une approche socioconstructiviste « en miroir » dans le sens où les futurs enseignants d'anglais doivent inscrire leur pratique de classe dans une approche communicative à visée actionnelle relevant de la même approche en relation avec l'apprenant et son milieu social.

\section{Mise en œuvre}

8 Le numérique a été le moyen utilisé dans ce projet. La fiche réalisée est aujourd'hui accessible aux inscrits de M1 MEEF (accès restreint). Dès la rentrée universitaire 2016-2017, nous avons fait appel au volontariat des fonctionnaires-stagiaires, leur demandant de proposer la trace écrite d'une séance (voire d'une séquence) représentative de leur activité de classe et développant un domaine langagier ou un outil méthodologique particulier. Les projets réceptionnés avaient tous des formes très hétérogènes parfois contre-productives. Nous les avons classés et avons tenté de leur donner une cohérence dans la forme et la présentation. Le résultat décevant nous a incitée à faire appel à un étudiant ${ }^{7}$ inscrit en Master $2^{\text {ème }}$ année de Pratiques et Ingénierie de la Formation (désormais PIF) afin de mettre en forme le matériel obtenu. Fin 2016, selon nos critères, il nous a fourni une fiche-type (voir annexe 2 « fiche-trame»). Elle a été amendée par les M2 MEEF (rubriques supplémentaires, présentation, etc.) ultérieurement, puis utilisée par le groupe en guise de trame, dès la rentrée de janvier 
2017. Elle comporte plusieurs rubriques : classe visée, durée de(s) (l')activité(s), objectifs, documents et supports. Chaque phase de la mise en œuvre pédagogique est ensuite décrite avec minutie. Il est aussi demandé à chacun de justifier ses choix didactiques. Le résultat final donne un matériel rationalisé et cohérent, accessible aux futur(e)s certifié (e)s et pouvant servir de référence ou de modèle, tout en étant conforme aux attentes du jury pendant le concours.

Prenons un cas précis. Une jeune fonctionnaire-stagiaire ${ }^{8}$ a proposé une séquence intitulée Halloween and Scary Times proposée sous forme de projet de classe (voir annexe 1). On constate dans ce travail spécifique que le style adopté relève plus de l'organisation personnelle et du suivi chronologique du plan de cours, que d'une composition finalisée dans un but d'auto-apprentissage. Quelques erreurs de langues sont à remarquer ainsi qu'un certain flou didactique, le tout rendant le document difficile à médiatiser en l'état. Aucune indication de timing des activités n'est indiquée, par exemple, ce qui manque aux étudiants de M1. Un travail sur les connecteurs logiques est avancé sans fiche de pratique raisonnée de la langue ou autre outil méthodologique. Faire état d'activités sans en produire d'exemple est assez anxiogène pour les candidats au concours. Sans modification, la fiche risquait d'être inutilisable. Nous avons alors décidé d'harmoniser la présentation générale de l'outil et de le standardiser afin de le rendre accessible au premier regard. Pour cela, il fallait repenser le travail pour lui donner cohérence et précision pré-professionnelle. Notre étudiant en PIF nous a alors proposé la «fichetrame» (voir annexe 2) que nous avons envoyée à l'ensemble des fonctionnairesstagiaires par le biais de la liste de diffusion de l'ESPE. À ce stade, nous avons proposé de ne relire que les outils «standardisés » et finalisés avant de les déposer sur la plateforme Moodle (voir annexe 6).

10 En annexe 3, on a le résultat de la fiche méthodologique revisitée et prête à être médiatisée. On constate que la première page est dédiée aux informations importantes à propos du projet mis en œuvre, soit la classe visée, le timing espéré, l'objectif rédigé en anglais ainsi que les documents exploités. Les étapes de la mise en œuvre suivent une chronologie pédagogique :

- introduire le thème abordé

- travailler le thème

- évaluer et synthétiser

11 Tout n'est pas parfait. Des précisions didactiques pourraient encore être apportées quant à la différence entre document et support, par exemple, ou bien quant à la rédaction de l'objectif en anglais langue-culture cible et la description des étapes. Les attendus de la séance (ou de la séquence) pourraient être rédigés suivant la formulation proposée dans la démarche didactique d'aujourd'hui, soit l'approche communicative à visée actionnelle : «l'élève sera capable de ». Cet ajout permettrait d'exploiter les critères et descripteurs des niveaux de compétence du CECRL ${ }^{9}$ et participer ainsi à la formation des M1. Nous avons d'ailleurs demandé à ce que le niveau visé soit mis en évidence en haut de la première page de façon à guider les futurs certifiés dans le projet didactique qu'ils vont défendre à l'oral du CAPES. L'évaluation devrait représenter une phase à part entière et non pas figurer avec la partie intitulée "synthétiser ». En effet, ce type d'activité relève d'un domaine langagier précis (oral ou écrit) et de compétences particulières (compétence ${ }^{10}$ pragmatique, par exemple) qui méritent d'être insérées dans les étapes mêmes du projet, dans la démarche d'apprentissage donc, en tant que tâche ${ }^{11}$ intermédiaire, par exemple. Des liens vers Éduscol ${ }^{12}$ pourraient également être insérés 
dans les modules afin de pouvoir se rendre directement sur le site et avoir accès aux programmes en langues vivantes, selon les attentes et projets pédagogiques mis en œuvre.

Beaucoup de ces modifications ont été apportées dans la dernière version du projet (voir annexe 4). Nous constatons que les niveaux CECRL visés sont à cocher, que l'évaluation a une place à part entière sur la fiche et que le lien vers Éduscol ( $Q R$ code) apparaît en haut à droite de la première page. À ce jour, nous n'avons pas modifié les 3 pages suivantes du travail. En effet, la question de la limite de notre expertise se pose ici. Doit-on modifier les projets proposés par nos jeunes collègues sous prétexte d'expérience personnelle ou doiton laisser chacun libre d'apprécier le travail pré-didactisé tel qu'il a été pensé et expérimenté sur le terrain ? Depuis février 2017, ces fiches-outil sont déposées sur la plateforme Moodle de l'ESPE et restreintes aux seuls inscrits en M1 MEEF (voir annexe 5).

Dans ce dispositif, notre rôle a été de mettre en relation une demande et sa réponse, de faire se rencontrer des étudiants inscrits dans le même parcours mais qui ne se connaissaient pas. Nous avons d'abord proposé une articulation M1/M2 avant d'organiser une rencontre en février 2017. Nous avons également servi de médiatrice entre les attentes des uns et les projets pédagogiques des autres. Pour tenter de répondre à notre problème, nous avons fait appel à un étudiant en ingénierie de l'éducation : il a été une ressource essentielle pour développer des «fiches-trames » et modéliser les documents. Enfin, nous avons relu chaque fiche avant sa diffusion, afin qu'elle réponde de façon raisonnable aux normes et objectifs fixés par le projet.

\section{Bilan et analyse}

Ce projet ayant pour finalité de numériser et médiatiser du matériel pédagogique élaboré par des étudiants de M2 comme aide à la préparation au concours des M1, semble proposer un travail transversal. En effet, si l'on inscrit l'approche pédagogique de l'enseignement supérieur dans une perspective communicative à visée actionnelle, le principe décrit ici propose bien d'apprendre à faire quelque chose (des outils pédagogiques) pour en faire quelque chose dans la vie (une tâche), soit préparer l'épreuve d'un concours en langue vivante, afin d'obtenir un poste de professeur. Cette perspective étant appliquée dans les pratiques de classe du second degré et dans l'enseignement supérieur, il y a là un effet de réciprocité propice à l'appropriation des savoirs et savoirs procéduraux pérenne. Depuis 2005, les fonctionnaires-stagiaires adossent leur pédagogie à l'approche actionnelle et ils doivent eux-mêmes accomplir une tâche non linguistique au service de leurs futurs collègues. Ils doivent prendre du recul par rapport à leur pédagogie afin de produire des modules pour leurs pairs de l'ESPE (M1) ce qui les incite à revoir certaines préparations ou mises en œuvre pédagogiques. En rédigeant leur fiche, ils vérifient la cohérence niveau CECRL visé/classe, le bienfondé des activités langagières dominantes, la cohérence des tâches et activités proposées, etc. Le lexique didactique est vérifié et adapté aux situations exploitées par nos soins. Les étudiants de M1 vont pouvoir utiliser ces travaux comme références pour préparer leurs séances de préparation à la MSP à l'université. Ces fiches représentent des cas concrets à exploiter et des exemples méthodologiques à réutiliser en cas de situations pédagogiques semblables ou comparables dans les dossiers travaillés en TD. Elles servent également de document de vérification en cas de doute à propos d'un niveau CECRL visé, d'une notion exploitée ou d'une tâche proposée. La présentation quasi-systématique imposée par la fiche-trame 
peut même servir de modèle pour préparer les épreuves d'admission au CAPES en juin. Les objectifs sont différents pour les M2. Élaborer de tels outils leur demande une réflexion et une démarche de type inductif sur leur travail. Ils doivent réfléchir à leur pratique professionnelle de façon à fournir des séquences exemplaires de qualité à leurs futurs collègues. L'étudiant en master PIF peut appliquer les enseignements reçus à l'ESPE à un projet précis et pratique. Ses connaissances techniques lui permettent de proposer un produit fini attractif et facile à utiliser de type responsive design apte à répondre aux demandes des utilisateurs. En tant qu'enseignante, nous avons un rôle d'intermédiaire dans ce projet. Nous agissons en tant que médiatrice mettant en relation les acteurs des deux années de Master mais laissons les différents protagonistes libres de leurs choix de supports et d'exploitations didactiques. Nous dispensons un enseignement frontal en présentiel (TD) et réceptionnons les projets qui doivent correspondre aux attentes des jurys de concours. Nous les examinons en tant qu'enseignante expérimentée, après plus de 20 ans passés dans l'enseignement secondaire. Les modules sont finalement transmis à notre interlocuteur futur spécialiste en outils numériques pour finaliser le projet.

Le temps passant, les étudiants se sont adressés directement à leur binôme de Master PIF. Ils ont pu ainsi expliquer certains aspects de leurs fiches, de façon à les insérer au mieux dans les documents, sans passer par le "pivot » ou l'enseignante de ESPE. Nous en avons tiré la conclusion qu'il serait intéressant, voire utile, de créer un forum de discussion sur la plateforme de l'École pour que les étudiants de Master 1ère année puissent librement s'entretenir avec leurs collègues de 2ème année, mais également avec l'enseignante. Ils pourraient alors avoir des précisions à propos d'une activité développée sur une fiche ou d'un domaine langagier privilégié lors d'une séance/séquence mais aussi sur le bienfondé d'une tâche finale, par exemple. Ces discussions techniques auraient lieu en dehors du temps universitaire, en fonction des disponibilités de chacun, de façon flexible.

Aujourd'hui, ce dispositif suit un mouvement unidirectionnel horizontal que nous pouvons représenter par le schéma suivant:

étudiants $\mathrm{M} 2(\mathrm{MEEF}) \longrightarrow$ enseignante $\longrightarrow$ étudiant $\mathrm{M} 2$ (PIF) $\longrightarrow$ étudiants $\mathrm{M} 1$ (MEEF)

16 À l'avenir, l'idéal serait d'impulser un mouvement de retour du type :

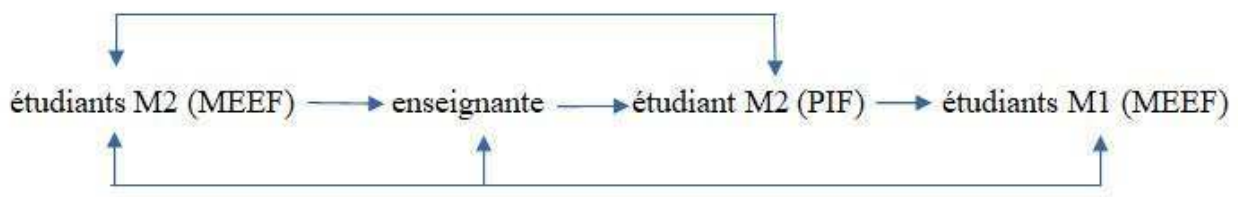

17 Le schéma nous montre que l'attitude de l'enseignant relève de celle du médiateur qui distribue les tâches et orchestre un projet mais également de l'expert. Aujourd'hui, l'autorité liée à l'âge ou à la hiérarchie laisse la place à la reconnaissance de la compétence et surtout à la manière de l'obtenir. Montrer comment savoir est devenu plus important que de monter que l'on sait. On transmet du savoir-faire et du savoir être aussi bien dans le secondaire que dans le supérieur dans le souci d'une formation tout au long de la vie. Les responsabilités sont partagées pour une formation centrée sur les valeurs et les compétences de chacun. Henri précise $(2011: 625)$ que ce type de médiation « n’est pas qu'une simple transaction ou qu'un compromis pratique. Elle implique une 
transformation profonde, la création de nouveaux liens et la rupture avec les structures constituées. »

Le contrat didactique enseignant/apprenant a été renégocié et la pédagogie partagée, voire mutualisée. La compétence d'enseignement au sens large est alors atteinte. En effet, selon Bourguignon (2015: 64) « la compétence est à la fois un ensemble de ressources, des savoirs et des savoir-faire et l'opérationnalisation de ces ressources [...]. »

Nous optons donc pour une "approche qualité » qui passe par la progression suivante (voir schéma ci-dessus) : besoin de terrain/systématisation/remise en cause/retour vers le terrain. Le savoir pédagogique est entre les mains des acteurs de terrain (M2) au service des apprenants (M1) expertisé par une enseignante et médiatisé par un étudiant en ingénierie de la formation. Collaboration et milieu interagissent. La formatrice n'a plus son rôle d'unique personne-ressource. Elle est le catalyseur des ressources disponibles. Elle rend les échanges possibles mais garde l'œil évaluateur de la référente experte. Elle relaie les acteurs de terrain, filtre les informations pour les redistribuer à bon escient et éliminer les parasites. Elle motive les M2 pour produire des documents et incite les M1 à les utiliser en citant les fiches dans son cours. Elle rappelle aux uns et aux autres les attentes inhérentes au projet de façon à respecter le calendrier imposé par les dates des épreuves orales du concours. Enfin, elle a des interactions avec ses étudiants de M1 et de M2 à propos de la pratique du métier d'enseignant qui aident les uns à préparer les épreuves orales du CAPES et qui soutiennent les autres dans leurs pratiques de classes respectives. Il y a alors communication horizontale entre étudiants (M2 et M1) plutôt que verticale entre professeure et étudiants.

L'étape suivante à ce projet va être la mise en place d'un forum où les étudiants pourront dialoguer à propos des outils proposés. Il est programmé pour la rentrée universitaire 2017-2018. Nous parlerons alors de co-construction. Dans cette situation, le statut de l'enseignant y perd peut-être en symbole d'autorité et de contrôle du savoir transmis, mais il y gagne en confiance et en crédibilité. Ce modèle remet en cause la transmission des savoirs et des savoir-faire de type local décrite par Morandi (2005: 111). L'apprentissage n'est plus contraint à une zone particulière de savoir mais peut être distant et asynchrone. Par contre, il s'insère dans un système global dans le sens où il prend place « dans un ensemble de ressources informationnelles pour le savoir » (op. cit.). L'apprentissage s'articule autour des besoins des étudiants par des actions sur mesure, sans cesse actualisées et mises à leur disposition par des moyens extérieurs : les technologies de l'information et de la communication (TICE). Ce mode de communication du savoir s'inscrit dans les objectifs de l'université en termes d'économie (ressources enseignantes supplémentaires) mais également dans une perspective nouvelle d'approche à la transmission dans l'enseignement supérieur. Elle correspond aux besoins de formation in situ tout au long de la vie des étudiants de M2 et agit au niveau de leur sentiment d'efficacité personnelle voire professionnelle (voir Bandura in Macré, 2014 : 122).

21 À ce jour, ce travail n'a permis de déposer que quelques fiches sur la plateforme Moodle de l'École (voir annexe 6). Le manque de temps disponible freine le projet. Nous réfléchissons à introduire ce projet de manière systématique à la rentrée universitaire prochaine. Il pourrait se transformer en application d'un des cours dispensés en présentiel intitulé « le développement des 5 activités langagières ", et éviterait l'abandon du processus. En effet, différentes études citées par Fenouillet et Déro (2006: 92) ont montré les limites de l'enseignement via différentes formes de technologies. 


\section{Conclusion}

À partir de cette expérience de terrain, nous avons voulu montrer comment répondre à la demande des apprenants de M1 en termes de formation (stratégie bottom up), en les impliquant dans l'élaboration d'un outil méthodologique spécifique. En effet, en partant d'un besoin émanant des étudiants préparant le CAPES d'anglais, inscrits en Master MEEF $1^{\text {ère }}$ année à l'ESPE de l'académie de XXX, des modules à visée didactique ont été élaborés. Ils ont été rédigés par les fonctionnaires-stagiaires inscrits en Master MEEF $2^{\text {ème }}$ année. Ils ont mis leur expérience d'ex-M1 au service des futurs certifiés de l'École, afin de les aider au mieux à la préparation des épreuves orales du concours du second degré, et plus particulièrement à l'épreuve de mise en situation professionnelle relevant de la didactique de l'anglais. Les candidats doivent proposer une mise en œuvre pédagogique permettant de travailler plusieurs activités langagières avec 1 voire 2 dominantes ainsi que des objectifs linguistiques et pragmatiques cohérents. D'autres acteurs sont intervenus dans ce projet de mutualisation et de partage de contenu : un étudiant inscrit en M2 PIF (partie numérique) ainsi que l'auteure de ces lignes (cohérence didactique). Ce travail autour des savoirs fondamentaux et des savoirs procéduraux (didactiser des documents et les adapter à ses classes) a donc fait se rencontrer et interagir des intervenants différents, des savoirs multiples différents ${ }^{13}$ et une technologie. Les tâches ont été fractionnées mais réalisées en fonction des compétences de chacun et mises au service de tous. Les M2 ont d'abord élaboré des séances ou des séquences didactiques non modélisées. Elles ont ensuite été adaptées au format de la fiche-trame avant d'être médiatisées. Le résultat - les modules pédagogiques - n'a été validé et déposé sur la plateforme Moodle de l'établissement qu'après être passé par le filtre de l'approche didactique en vigueur aujourd'hui dans les classes, soit l'approche communicative actionnelle. Elle fait le lien avec les épreuves du CAPES et la pédagogie institutionnelle.

Ce dispositif a pour finalité de mutualiser les travaux des étudiants tout en travaillant sur leur motivation et la qualité pédagogique de la formation dispensée. Ces fiches sont consultables sur différents supports (téléphones, tablettes, ordinateurs) grâce à un $\mathrm{QR}$ code de façon à pouvoir travailler de manière totalement asynchrone.

\section{BIBLIOGRAPHIE}

Bailly, D. (1997). Didactique de l'anglais. Objectifs et contenus de l'enseignement. Paris : Nathan.

Beguin, N. \& Garcia, C. (1996). La séquence didactique an anglais. CRDP Midi-Pyrénées : Bertrnadlacoste.

Bonnet, F. et alii. (2012). Réussir l'épreuve de leçon au CAPES d'anglais. Bordeaux : Presses Universitaires de Bordeaux.

Bourguignon, C. [(2005) 2015]. La démarche didactique en anglais. Du concours à la pratique. Paris : PUF.

Recherches en didactique des langues et des cultures, 16-1 | 2019 
Brunot, R. \& Grosjean, L. (1999). Apprendre ensemble. Pour une pédagogie de l'autonomie. CRDP de l'Académie de Grenoble.

Dewey, J. (1976 [1945]). L'école et l'enfant. (L.S. Pidoux trad.). Neuchâtel : Delachaux et Niestlé.

Éduscol : www.eduscol.education.fr (consulté le 10-03-2017).

Fenouillet, F., Déro, M. (2006). « Le e-learning est-il efficace ? Une analyse de la littérature anglosaxonne ». Revue Savoirs, vol. 3, $\mathrm{n}^{\circ} 12$. pp. 88-101.

Guichon, N. \& Tellier, M. (dirigé par) (2017). Enseigner l'oral en ligne. Paris : Didier.

Henri, F. (2011). « Où va la distance ? est-ce la bonne question?». Revue Distances et savoirs, vol. 9, nº. pp. 681-630.

Julié, K. \& Perrot, L. (2008). Enseigner l'anglais. Paris : Hachette Éducation.

Lamy, M.-N. (2011). « Où va la distance, par des temps agités ? Réponses d'une organisation distancielle, l'Open University ». Revue Distances et savoirs, vol. 9, n4. pp. 611-618.

Lions-Olivieri, M.-L. \& Liria, P. (2009). L'approche actionnelle dans l'enseignement des langues. Paris : Maisons des Langues Édition.

Macré, N. (2014). « Les apprenants LANSAD de niveau A2/B1 en première année d'anglais en autonomie accompagnée médiatisée : quels besoins ? ». Revue Cahiers de l'APLIUT, vol.33, n², pp. 118-141.

Martin, C. (2015). «Les dispositifs hybrides : l'avenir du cours de langue au niveau master?». Revue Cahiers de l'APLIUT, vol. 34, $\mathrm{n}^{\circ} 1$. pp. 166-180.

Ministère de l'Éducation nationale.http://www.devenirenseignant.gouv.fr/cid98575/lesepreuves-du-capes-externe-et-du-cafep-capes-section-langues-vivantes-etrangeres.html.

Morandi, F. (2005 [1997]). Modèles et méthodes en pédagogie. Paris : Nathan.

Narcy-Combes, M.-F. (2005). Devenir professeur de langue. Paris : Ellipses.

Piaget, J. (1969). Psychologie et pédagogie. Paris : Denoël.

Pinéda, I. (2016). La didactique au CAPES d'anglais. Paris : Ophrys.

Quivy, M. \& Tardieu, C. (1999). Réussir l'épreuve de didactique à l'agrégation interne. Paris : Ellipses.

Rivens Mompean, A. (2013). Le Centre de Ressources en Langues : vers la modélisation du dispositif d'apprentissage. Villeneuve d'Ascq : Presses Universitaires du Septentrion.

Rivière, R . (1990). La psychologie de Vytgosky. Liège : Pierre Mardaga Éditeur.

Stark, J. et alii. (2011). Meeting Point $1^{\mathrm{èr}}$. Paris : Hatier.

Tagliante, C. ([1994] 2001). La classe de langue. Paris : CLE international.

Vigneron, D. Vandewoude, D. \& Pineira-Tresmontant, C. (2015). L'enseignement-apprentissage des langues étrangères à l'heure du CECRL. Arras : Artois Presses Université.

Whyte, S. (2014). « Bridging the gaps: Using social media to develop techno-pedagogical competences in pre-service language teacher education ». Revue Cahiers de l'APLIUT, vol. 33, $\mathrm{n}^{\circ} 2$, pp.143-169. 


\section{ANNEXES}

\section{Annexe 1 : projet de classe Halloween and Scary Times}

Quatriéme - Séquence 3: Halloween and scary times

Tâche finale : write and tell a scary story to the class during our Halloween hou

Séquence assez courte ( sur deux semaines, 6heures) avant les vacances d'automne.

Premiéres séances d'introduction dans le thème d'Halloween : les traditions, comment le fête-on puis apport lexical à partir du clip Thriller de Michael Jackson. Distribution de ' my scary dictionary' : outil qui sera à remplir au fur et à mesure de la séquence afin de leur scary dictionary' : outil qui sera à remplir au fur et a mesure de

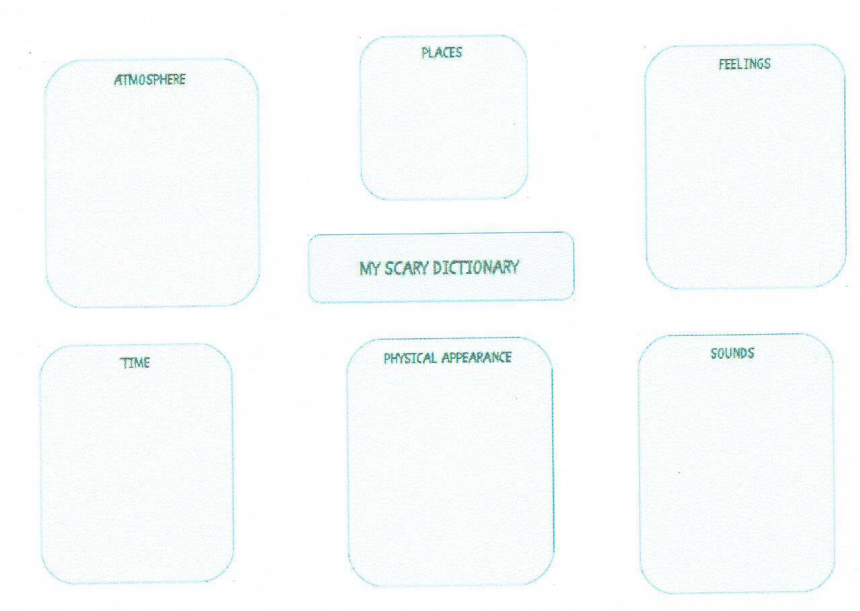

Travail sur la création d'une histoire, étude d'un extrait de Roald Dahl, The BFG : les élèves doivent remettre l'histoire dans l'ordre et lors de la mise en commun nous cherchons ensemble les indices qui les ont aide.

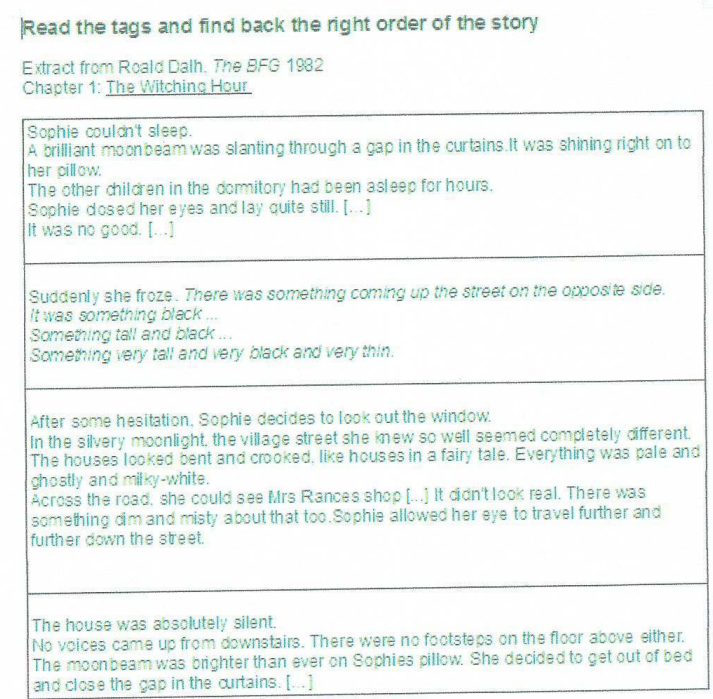

Aprés ça, reflexion sur la construction d'une histoire à l'aide de la fiche « how to write a scary story » : nous permet de dégager les différentes étapes et le contenu d'une histoir (+ travail sur les connecteurs logiques et adverbes utiles dans un récit : suddenly..) (travail sur Con confondre les genres pr réussir la tache finale. 


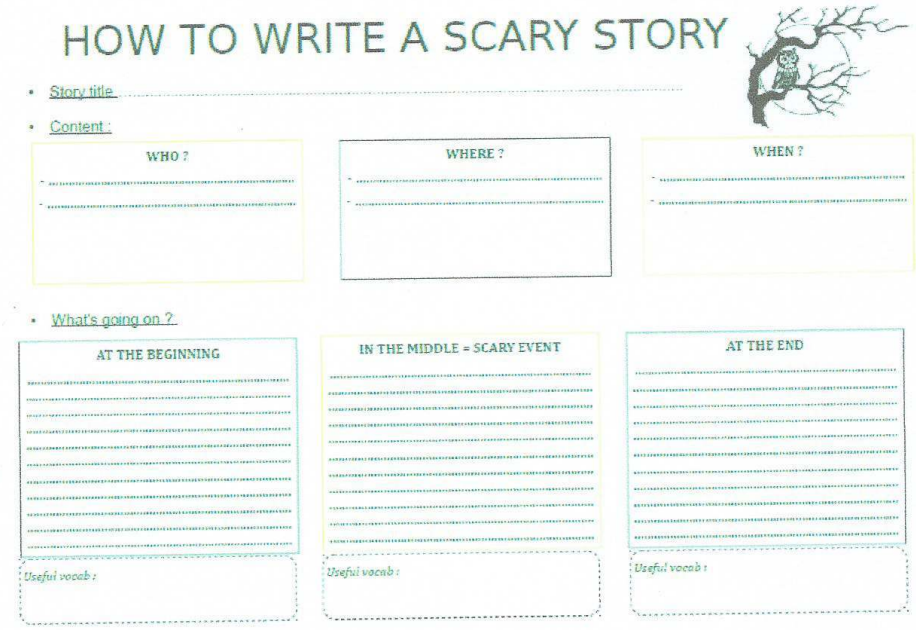

PRL réactivation sur le préterit ( temps à utiliser lors de la rédaction de leurs histoires).

Heure de rédaction, possibilité d'avoir des fiches « how to write a scary story " vierges afin de les compléter avec leurs propres idées qu'ils auront juste à assembler pour créer leurs textes

Les histoires seront à la fois notés en tant que productions écrites mais aussi comme productions orales car le but est de faire vivre leurs idées et d'effrayer leurs camarades. + vote pour la meilleure histoire : to win your Halloween treat..

\section{Annexe 2 : « fiche-trame »}
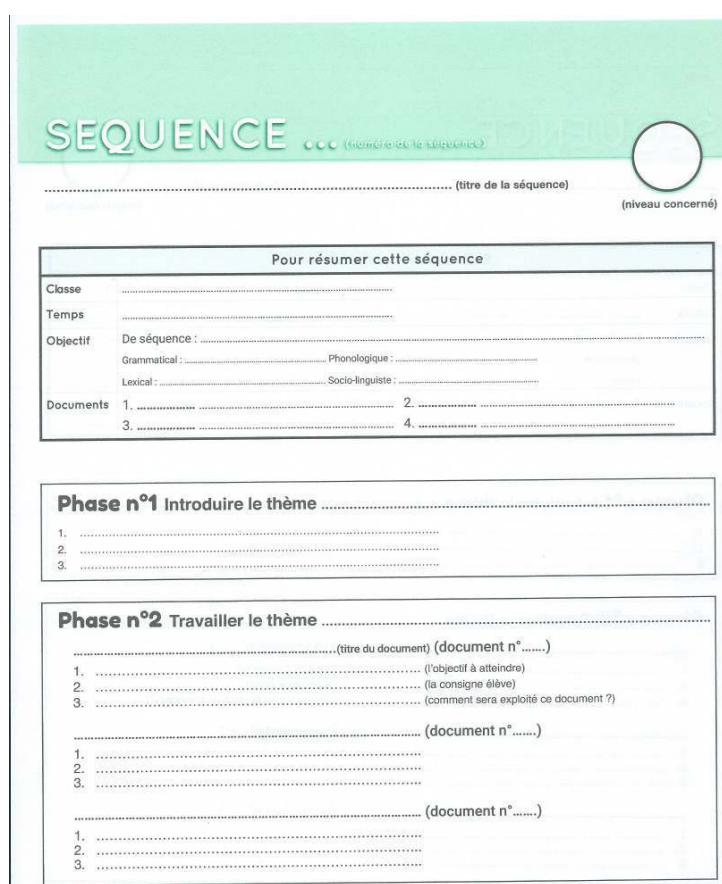

Phase $n^{\circ} 3$ Évaluer et synthétiser (cocher les options)

1. Production $\square$ ècrite $\square$ orale, $\square$ avec la possibilité de disposer de ...............................................

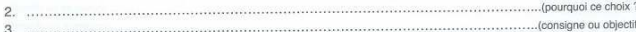


Annexe 3 : « fiche-outil » réalisée à partir du projet de classe Halloween and Scary Times (4pages). Première version.

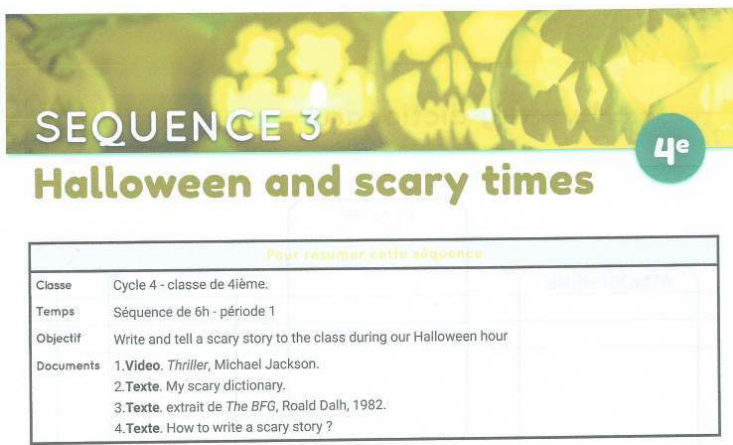

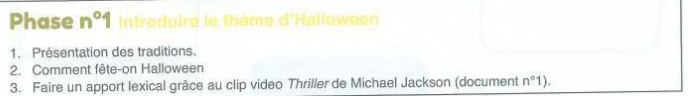

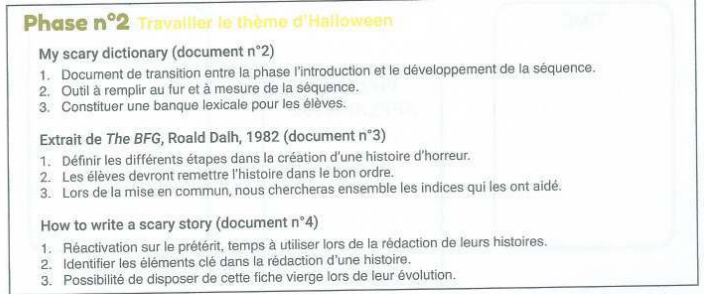

Phase $n^{\circ} 3$ Evaluer et synthitiser ser
1. Production écrite, avec la possibiite de disposer de la fiche How to write a scary story vierge.
2. Production orale, puisque le but est de faire vivre leurs idées en essayant d'effrayer leurs camarades.
3. Voter pour la meilleur histoire d'horreur : to win your Halloween treat...

Annexe 4 : « fiche-outil » réalisée à partir du projet de classe Halloween and Scary Times. Seconde version, page 1.

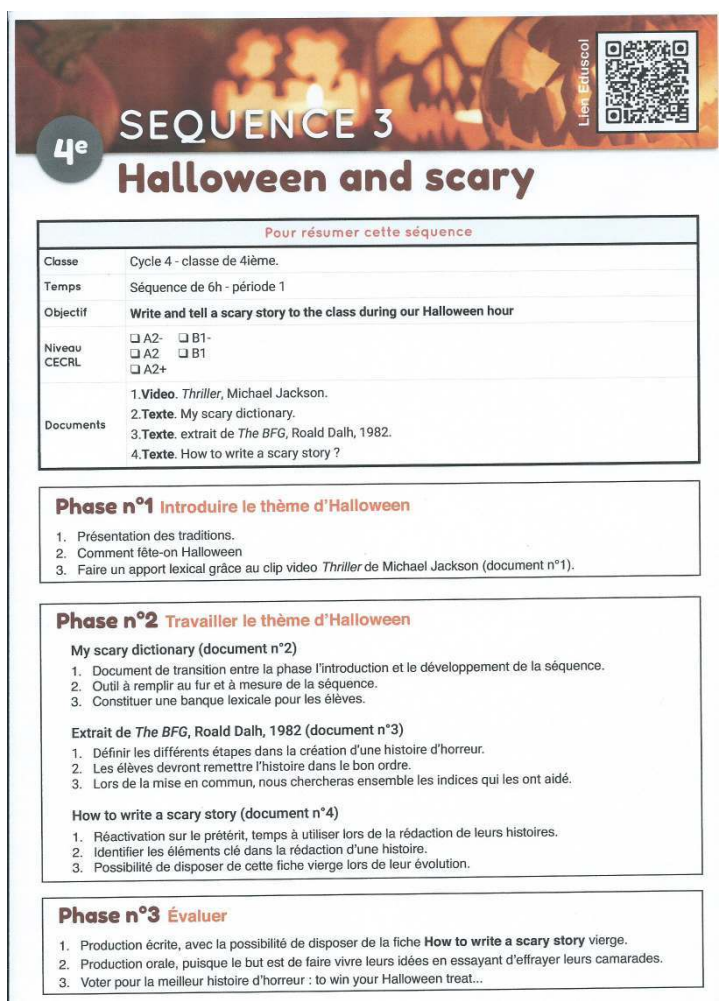


Annexe 5 : lien vers les modules sur la plateforme de l'École.

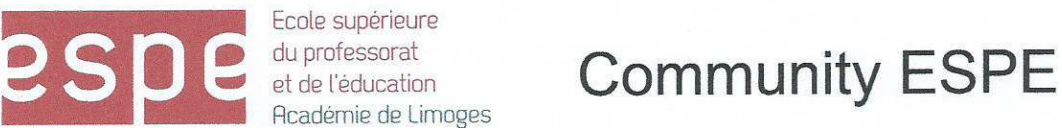

Accueil / Cours / * Master MEEF Professeur de Langue (Allemand, Angl...

/ 2016-2017 Parcours Professeur d'Anglais

Full screenclose full screen

Catégories de cours: * Master MEEF Professeur de Langue (Alle

Rechercher des cours

Valider

2016-2017-M1-M2 Anglais outils pédagogiques

outils pédagogiques pour les M1 MEEF anglais

aide à la préparation des oraux du CAPES (didactique)

Cours $\Rightarrow$

Annexe 6 : tableau récapitulatif des fiches finalisées

\begin{tabular}{|l|l|l|}
\hline Titre & Classe concernée & Niveau CECRL visé \\
\hline Uniforms & $6 \mathrm{e}$ & A2 \\
\hline Halloween and Scary Times & $4 \mathrm{e}$ & A2/B1 \\
\hline Going Abroad & $3 \mathrm{e}$ & B1 \\
\hline Australian Wildlife & 2nde & A2/B1 \\
\hline The London Dungeon & 2nde & A2/B1 \\
\hline Brown vs. Board of Education (2011:90) & 1ère ES & B1/B2 \\
\hline The CIA has a new “panopticon"14 & 1ère ES & B1/B2 \\
\hline
\end{tabular}

\section{NOTES}

1. Moodle est une plateforme d'apprentissage en ligne qui sert à créer des groupes autour de contenus et d'activités pédagogiques.

2. Interrogations des étudiants dans les conditions du concours.

3. Cet étudiant a verbalisé les besoins de la façon suivante: «nous ne rencontrons les M2 que pendant le conseil de perfectionnement. Et là nous avons des tas de choses à nous dire. Il faudrait le faire plus souvent, ça nous serait utile pour la suite». 
4. Consulter le site officiel : http://www.devenirenseignant.gouv.fr/cid98575/les-epreuves-ducapes-externe-et-du-cafep-capes-section-langues-vivantes-etrangeres.html.

5. Bourguignon (2015: 119) nous explique que la seconde partie en langue française consiste « en la proposition de pistes d'exploitation didactiques et pédagogiques de ces documents, en fonction des compétences linguistiques qu'ils mobilisent, de l'intérêt culturel et de civilisation qu'ils présentent, ainsi que des activités langagières qu'ils permettent de mettre en pratique selon la situation d'enseignement choisie, suivie d'un entretien en français au cours duquel le candidat est amené à justifier ses choix. »

6. Un stage massé est limité dans le temps. Dans le second degré il est de deux semaines consécutives par opposition au stage filé qui a lieu de façon régulière dans l'année le lundi, par exemple.

7. Nous tenons à remercier XXX (M2 PC PIF) pour son aide et sa disponibilité. Sans ses conseils avisés et son travail régulier ce projet n'aurait pas abouti.

8. Nous remercions XXX (M2 FSTG MEEF) pour sa proposition de séquence.

9. CECRL: Cadre Européen Commun de référence pour les langues. Des niveaux visés correspondent aux attentes par cycle.

10. «Les compétences sont l'ensemble des connaissances, des habiletés et des dispositions qui permettent d'agir. » CECRL (15). « De manière générale, une compétence est la mobilisation d'un ensemble de savoirs et de savoir-faire organisés en vue d'accomplir une activité complexe ou une tâche. » Bourguignon (2015:63).

11. "Est définie comme tâche toute visée actionnelle que l'acteur se représente comme devant parvenir à un résultat donné en fonction d'un problème à résoudre, d'une obligation à remplir, d'un but qu'on s'est fixé. » CECRL (16).

12. Site de l'Éducation nationale.

13. Il s'agit de savoirs didactiques en pré-construction pour les M1 et de savoirs en construction pour les M2.

14. Document adapté par l'enseignant fonctionnaire-stagiaire du New York Times, Steve Lohr et Katie Benner, "With WikiLeaks Claims of C.I.A. Hacking, How Vulnerable Is Your Smartphone ?" 7 mars 2017.

\section{RÉSUMÉS}

Dans cet article, nous proposons de décrire la mise en place d'un outil pédagogique intégré à la formation des futurs enseignants d'anglais dans le second degré au sein de l'ESPE de l'académie de XXX.

Le but visé est de proposer des travaux déjà expérimentés par les fonctionnaires-stagiaires aux étudiants préparant les épreuves d'admission du concours externe du CAPES, par le biais de la plateforme Moodle de l'établissement. La numérisation est assurée par un étudiant de l'École inscrit en Master PIF.

Cette expérience relève de la médiation enseignant/stagiaire/étudiant. Elle se situe de manière transversale entre l'intervention directe du formateur en relation avec les futurs certifiés, mais également avec les jeunes professionnels conscients des exigences du concours.

outil pédagogique, plateforme d'apprentissage en ligne, ESPE, didactique de l'anglais, CAPES, fonctionnaires-stagiaires, Master PIF 
This article focuses on the development of a pedagogical tool integrated into the training of future secondary education teachers of English in XXX Superior School of Education.

The aim is to provide class works that have already been carried out in classes by in-service teachers to pre-service students preparing the oral tests included into their final competitive exam.

The device is to be used online (LMS) after having been digitized by a School student in Education Engineering.

This experiment explores the teacher/trainee/student relationship. Thus it questions the university teacher face-to-face lectures compared to a collaborative pre-service student training.

\section{INDEX}

Keywords : pedagogical tool, LMS, Superior School of Education, applied linguistics in English, competitive exam in Education and Training Professions, in-service students, Master's degree in Education Engineering

Thèmes : Articles pédagogiques

\section{AUTEUR}

\section{SOPHIE DUFOSSÉ SOURNIN}

ESPE de l'académie de Limoges

Sophie Dufossé Sournin est maître de conférences à l'ESPE de l'académie de Limoges et membre du CeReS (EA 3648). Ses recherches en didactiques de l'anglais couvent la pratique de classe des futurs enseignants de langues vivantes étrangères, l'élaboration d'outils à mettre à la disposition des étudiants non-spécialistes de l'anglais (secteur LANSAD) et la certification de l'enseignement supérieur. Elle est actuellement directrice adjointe de l'ESPE (École supérieure du professorat et de l'éducation) de l'académie de Limoges en charge des relations avec les corps d'inspection et les EPLE et directrice du département de langues vivantes.

sophie.dufosse@unilim.fr 